\title{
Statistical Entropy of Radiation: Different Approaches
}

\author{
A.V. Shepelev \\ I.M. Gubkin Russian State University, Department of Physics, Moscow, Russia 119991 \\ *Corresponding Author: shepelev@rambler.ru
}

Copyright (C) 2013 Horizon Research Publishing All rights reserved.

\begin{abstract}
A number of definitions of entropy in optics have both advantages and disadvantages. The major features of different entropy definitions are briefly characterized. The principal distinction between the statictics entropy definition and the thermodynamics one is indicated. The diffraction-caused radiation entropy is calculated.
\end{abstract}

Keywords Optics, Radiation, Entropy, Thermodynamics

PACS 78.20.Bh

\section{Introduction}

According to the memoirs C. Shannon, when discussing the ideas that formed the basis of his famous work [1], John von Neumann told him: "You should call it entropy, for two reasons ... In the second place, and more important, nobody knows what entropy really is". Now a number of problems in quantum and statistical optics requires the concept of radiation entropy, but at the moment there are a lot of different entropy definitions. All of them have both advantages, and drawbacks. Characterising them, first of all it is necessary to distinguish the entropy introduced through concepts of warmth and temperature, i.e. thermodynamically (R. Clausius), from the entropy determined through the concept of distribution function, the field of physics where the first were L. Boltzmann and J.W. Gibbs.

Only the most known and often used for description of the optical phenomena the entropy concepts presented by different authors are considered in this short review. More information about the problem of radiation entropy and specific results can be obtained from the referenced papers and their references.

\section{Results}

1) Planck's entropy of radiation [2] is based on the concept of radiation brightness $B$ and on the distribution function of a system of noninteracting bosons:

$$
S_{P L}=\frac{k v^{2}}{c^{2}}\left\{\left(1+\frac{c^{2} B}{h v^{3}}\right) \ln \left(1+\frac{c^{2} B}{h v^{3}}\right)-\frac{c^{2} B}{h v^{3}} \ln \frac{c^{2} B}{h v^{3}}\right\} .
$$

It can be shown, that Planck's entropy of the black radiation is proportional to the third degree of temperature.

Unlike other entropy definitions in classical physics, such as Boltzmann's entropy, the Planck's entropy can be used even in case if the measure, including a Lebesgue's measure, is not conserved. Therefore Planck's entropy is applicable to the irreversible processes which do not obey the Liouville's theorem.

Besides fundamental importance of Planck's radiation entropy for the solution of blackbody radiation problem, that has led to occurrence of quantum physics, it is possible to note also only some particular results:

The general thermodynamic restrictions on efficiencies of the Stokes and anti-Stokes luminescence have been established [3]; the general conditions on radiation temperature rising in passive optical systems have been received, that, in particular, has allowed interpreting the features of nature of astrophysical masers. [4,5].

However Planck's entropy is based on concept of brightness which is not an observable value [6]. First, for brightness calculation, both the knowledge of local values of coordinate and the knowledge of a corresponding projections of radiation impulse are necessary simultaneously, that is impossible. Secondly, there is no exact definition of brightness of partially coherent radiation. Its definition through coherence degree and Wigner's function,

$$
B(\mathrm{r}, \mathrm{n})=\frac{k}{2 \pi} \int W\left(\mathrm{r}+\mathrm{r}^{\prime} / 2, \mathrm{r}-\mathrm{r}^{\prime} / 2\right) \exp \left(-i k \mathrm{~s}_{\perp} \mathrm{r}^{\prime}\right) d^{2} \mathrm{r}^{\prime}
$$

( $W$ is the cross-spectral dencity function in points $\mathrm{r}+\frac{\mathrm{r}^{\prime}}{2}, \mathrm{r}-\frac{\mathrm{r}^{\prime}}{2}, k$ is a wavenumber, $\mathbf{n}$ is a unit directional vector) is more heuristic, than proved.

2) Shannon's entropy is one of the fundamental concepts of the classical information theory, and is defined as: $\sum p_{i} \ln p_{i}$, where $p_{i}$ is probability. However for noncommuting operators, in particular for operators of coordinate and impulse, it is impossible to consider a joint distribution of corresponding observables. In quantum optics, a numer of authors propose to account additively the contribution of coordinate and impulse distributions into this entropy, 


$$
S_{S H}=-\int|\psi(x)|^{2} \ln |\psi(x)|^{2} d x-\int|\psi(p)|^{2} \ln |\psi(p)|^{2} d p
$$

The fundamental inequality, named the entropic uncertainty relation, is proved for such presentation of entropy:

$$
S_{S H} \geq 1+\ln \pi
$$

For recently offered probability representation of quantum mechanics other new inequalities for distributions of probabilities have been received also [7]. It is shown, that this entropy correlates with squeezing and other nonclassical characteristics of radiation states [8].

(Particular subject is the use of the maximum entropy method (MEM) for correcting the optical information. A lot of works is devoted to this subject. Application of MEM enables to increase significantly the resolving power of an optical system. Usually, the entropy of an optical image is calculated according to Shannon, but in some cases it is preferable to use the Boltzmann's entropy.)

3) Wehrl's entropy is defined via coherent states [9],

$$
\alpha=\frac{1}{\sqrt{2 \hbar}}(q+i p), \quad \rho_{W}(\alpha)=\langle\alpha|\hat{\rho}| \alpha\rangle,
$$

as

$$
S_{W}=S\left(\rho_{W}(\alpha)\right)=-\frac{1}{\pi} \int \rho_{W} \ln \rho_{W} d^{2} \alpha .
$$

So, the Wehrl's entropy is based on the quasi-probability, not on the probability itself. Obviously, according to uncertainty principle, $\rho(\alpha)$ cannot by localized within the area that is smaller than $h$. (P. Erenfest was the first who suggested the idea of introduction of the similar entropy, that accounted for a minimal possible phase volume).

For Wehrl's entropy the condition of monotony for compound systems is satisfied: if a system $A$ is a composition of two subsystems 1 and 2 , then

$$
S_{W}(A) \geq S_{W}(1) .
$$

The mimimum value of the Wehrl's entropy is 1, unlike Neumann's entropy and Shannon's entropy. For coherent states, $S_{W}=1$.

Wehrl's entropy describes well enough also the time dynamics of systems, for example time evolution of the isolated system "a field + two-level atom" [10]. Wehrl's entropy in this example approaches saturation, though not monotonically in time.

For photon-number states radiation the Wehrl's entropy is given by [10]:

$$
S_{W}=1+n+\ln n !+\gamma n-n \sum_{k=1}^{n} \frac{1}{k} .
$$

4) Von Neumann's entropy

$$
S_{N}=-k \operatorname{Tr}(\rho \ln \rho),
$$

expressed through a density matrix, is widely used in quantum mechanics. Moreover, in many respects this concept has formed a basis of rapidly developing area of mathematics - non-additive theory of probability. The Von Neumann's entropy of the whole system can be zero, but the entropy of the sybsystems differs from zero. Statistical distribution arises in this case, as it were, out of nothing.

However von Neuman's entropy contradicts the thermodynamic entropy increasing with time up to the greatest possible value corresponding to an equilibrium condition. From the movement equation for a density matrix

$$
i \hbar \frac{\partial \rho}{\partial t}=[H \rho]=H \rho-\rho H
$$

and definition of von Neumann's entropy, the movement equation follows for entropy

$$
-i \hbar k^{-1} \frac{d S_{N}}{d t}=\operatorname{Tr}(H \rho \ln \rho-\rho H \ln \rho)
$$

From the property of a trace commutativity,

$$
\operatorname{Tr} \rho H \ln \rho=\operatorname{Tr} H(\ln \rho) \rho
$$

and from commutativity of the density operator $\rho$ and its function $\rho \ln \rho$, it follows, that

$$
\operatorname{Tr} H \rho \ln \rho=\operatorname{Tr} \rho H \ln \rho \quad \text { i.e. } \quad \frac{d S_{N}}{d t}=0
$$

Hence, the von Neumann's entropy of a closed system remains constant in time (more detailes are in [11]). That is why the von Neumann's entropy in quantum optics does not enable to get a correct answer for the irreversible processes. Thus, the quantum entropy fails when one wants to use it for justifying the classical entropy. But if excluding the variables, which concern the parameters of matter, conversion of the incident-field characteristic function to the scattered-field one is not unitary, and can not be described by the Schrödinger's equation, but can be described by the kinetic equation. Only in this case the von Neumann's entropy is not constant for a closed system and can coinside with the thermodynamics entropy.

5) As for example it is useful to consider the increase of the radiation entropy due to a finite beam aperture. Localization of radiation by a finite aperture leads to uncertainty of the wave vector and, consequently, to emergence of the irreversibility. As mentioned above, it can be adequately described only by means of the thermodynamics entropy of radiation introduced by Planck. (For the equilibrium radiation, the von Neumann's and Planck's entropies actually coincide under replacement of the normalized brightness by the number of photons per mode $n$, and are defined by the equilibrium distribution of the non-interacting bosons: $\left.S_{N}=\int_{\Omega} \int_{\sigma}\{(1+n) \ln (1+n)-n \ln n\} d \Omega d \sigma\right)$.

For axially symmetric, generally "soft" aperture, both the 
brightness and the number of photons per mode are proportional to $U^{2} D$. Here, $U 2$ is the intensity distribution over the aperture, $D$ is the directivity pattern in the far-field zone. The latter is expressed by the Hankel transform:

$$
\begin{gathered}
F_{q}=\frac{1}{(2 \pi)^{2}}\left(\int U(r) I_{0}(q r) r d r\right) \\
D(q)=\frac{F_{q}^{2}}{\int F_{q}^{2} q d q}
\end{gathered}
$$

This allows calculate the thermodynamics entropy of the radiation diffracted by the aperture, or, equivalently, of the radiation emitted by the antenna with the same intensity distribution. (In order to make adequate the comparison between the entropies of radiation diffracted by different apertures, the additional conditions have been imposed - the equality of radiation power and the ideal transmission at some point). Fig.1 shows the entropy of the transmitted radiation, depending on the ratio of the effective diameter of the aperture to the wavelength for a circular hole and a Gaussian aperture. For both apertures there is a monotonic decrease of entropy with increasing of this ratio, which is explained by the decrease of the diffraction effects. The generalization to non-monochromatic radiation follows from the additivity of entropy for radiation with thermal statistics.

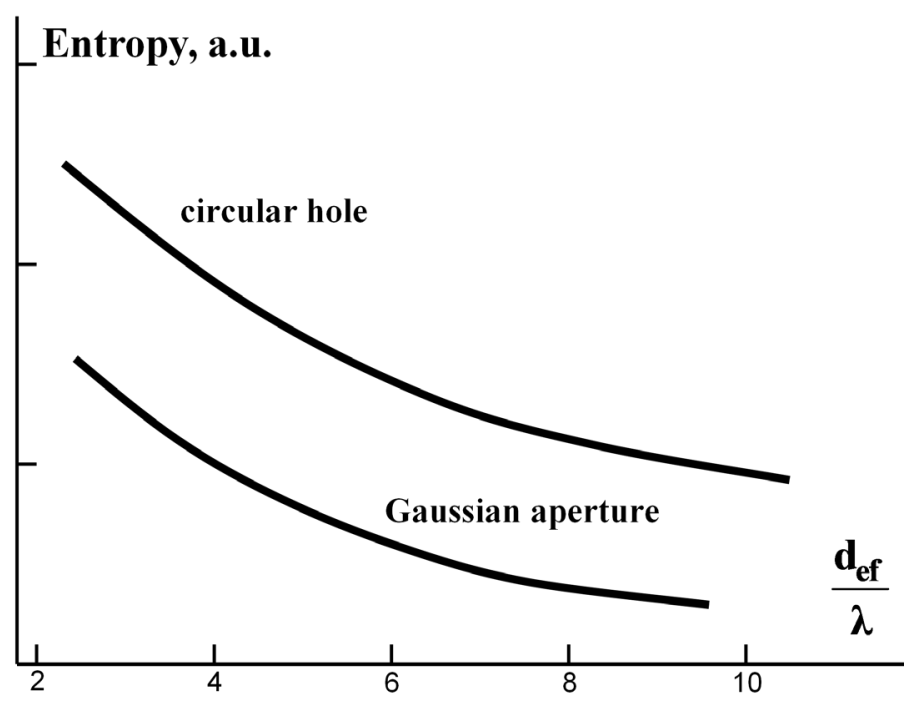

Figure 1. Entropy of the diffracted radiation depending on the ratio of the effective aperture diameter to a wavelength

\section{Conclusions}

Only just some of the most known definitions of entropy of radiation are described above. There are other ones: Kolmogorov's entropy, Renyi entropy, Wigner-Yanase-Dyson "skew entropy", etc. All of them can give different results when applied to the same problem. The detailed comparison of all of them can be done only within a large-space review book. The main feature of all entropy definitions, which were considered in this short paper, is that it is impossible to state that any of them adequately satisfy the condition of thermodynamic irreversibility. The main reason is that these attempts are actually based either on maintaining the Lebesgue's measure in the Liouville's theorem, or on a unitarity of the transformation - it is a reflection of the fact that all the laws of physics (with the exception of the second law of thermodynamics) are reversible in time. The most important is that the thermodynamics definition of entropy characterizes the irreversibility, whereas the statistics one defines the degree of disorder.

Thus, one can consider the von Neumann's opinion, devoted abowe and being said about sixty five years ago, to be valid for the entropy of radiation up to now.

\section{REFERENCES}

[1] C. Shannon. Bell System Technical Journal 27379 (1948).

[2] M. Planck. Annalen der Physik 16818 (1901).

[3] L.D. Landau. Journal of Physics USSR 10508 (1946). 
[4] A.V. Shepelev. Monthly Notices of the Royal Astronomical Society 378753 (2007).

[5] A.V. Shepelev. American Journal of Physics 78158 (2010).

[6] E. Wolf. Journal of the Optical Society of America 686 (1978).

[7] S. De Nicola, R. Fedele, M.M. Man'ko and V.I. Man'ko. Theoretical and Mathematical Physics 152 1081 (2007).

[8] A. Orlowski. Phys. Rev. A 56 (1997) 2545.

[9] A. Wehrl. Reports on Mathimatical Physics 16353 (1979).

[10] A. Orlowski. Phys. Rev. A 48727 (1993).

[11] N.N. Bogolubov and N.N. Bogolubov, Jnr. Introduction to Quantum Statistical Mechanics (Gordon and Breach, 1992). 